\title{
Chapter 11 \\ Impact of Electricity Market Designs on Investments in Flexibility Options
}

\author{
Christoph Fraunholz, Andreas Bublitz, Dogan Keles, and Wolf Fichtner
}

\begin{abstract}
Against the background of several European countries implementing capacity remuneration mechanisms (CRM) as an extension to the energy-only market (EOM), this chapter provides a quantitative assessment of the long-term crossborder effects of CRMs in the European electricity system. For this purpose, several scenario analyses are carried out using the electricity market model PowerACE. Three different market design settings are investigated, namely, a European EOM, national CRM policies, and a coordinated CRM. The introduction of CRMs proves to be an effective measure substantially shifting investment incentives toward the countries implementing the mechanisms. However, CRMs increase generation adequacy also in the respective neighboring countries, indicating that free riding occurs. A coordinated approach therefore seems preferable in terms of both lower wholesale electricity prices and generation adequacy.
\end{abstract}

\subsection{The European Debate on Electricity Market Design ${ }^{1}$}

Since the liberalization of the electricity markets in the 1990s, the prevailing market design in European countries has been the energy-only market (EOM), in which capacity providers are solely compensated for the amount of electricity they sell on the markets. In this market design, according to theory, scarcity periods lead to peak prices, which enables investors to cover their fixed and capital costs. In other regions of the world, e.g., in several US markets, so-called capacity remuneration mechanisms (CRMs) are a common extension of the EOM with the earliest implementations dating back to the late 1990s (Bublitz et al. 2019). These mechanisms typically aim to reduce the investment risks by offering capacity providers supplementary income on top of the earnings from selling electricity on the spot markets.

\footnotetext{
${ }^{1}$ This introductory section was previously published in Fraunholz and Keles (2019).

C. Fraunholz $(\varangle) \cdot$ A. Bublitz $\cdot$ D. Keles $\cdot$ W. Fichtner

Institute for Industrial Production (IIP), Karlsruhe Institute of Technology (KIT), Karlsruhe,

Germany

e-mail: Christoph.Fraunholz@kit.edu
} 
The additional generation, storage, or demand side capacity may then in turn help to improve generation adequacy, i.e., avoid shortage situations. Following the classification of the European Commission (2016), six generic types of CRMs can be distinguished:

- Tenderfor new capacity. Financial support is granted to capacity providers in order to establish the required additional capacity. Different variations are possible, e.g., financing the construction of new capacity or long-term power purchase agreements.

- Strategic reserve. A certain amount of additional capacity is contracted and held in reserve outside the EOM. The reserve capacity is only operated if specific conditions are met, e.g., a shortage of capacity in the spot market or a price settlement above a certain electricity price.

- Targeted capacity payment. A central body sets a fixed price paid only to eligible capacity, e.g., selected technology types or newly built capacity.

- Central buyer. The total amount of required capacity is set by a central body and procured through a central bidding process so that the market determines the price.

- De-central obligation. An obligation is placed on load-serving entities to individually secure the total capacity they need to meet their consumers' demand. In contrast to the central buyer model, there is no central bidding process. Instead, individual contracts between electricity suppliers and capacity providers are negotiated.

- Market-wide capacity payment. Based on estimates of the level of capacity payments needed to bring forward the required capacity, a capacity price is determined centrally, which is then paid to all capacity providers in the market.

In recent years, several European countries seem to face threats in terms of the future generation adequacy and therefore have either already implemented some kind of CRM or are currently in the process of evaluating appropriate solutions (cf. Fig. 11.1). These developments can be attributed to a variety of factors including strongly increasing shares of fluctuating electricity generation from renewable energy sources (RES), decreasing wholesale electricity prices as well as recent phase-out decisions for certain technologies. Yet, the tendency toward applying CRMs to increase investment incentives contradicts the European Commission's preference for an EOM in order to trigger new investments and provide signals for decommissioning in case of overcapacities. Moreover, in a highly interconnected electricity system like the European one, the uncoordinated implementation of local mechanisms might lead to potentially adverse cross-border effects, which stands in strong contrast to the European Commission's goal of creating an internal electricity market in Europe (Bublitz et al. 2019).

This chapter therefore aims to provide a quantitative assessment of the long-term cross-border effects of CRMs in the European electricity system. The electricity market model PowerACE is applied to a region covering Central Western European 
Fig. 11.1 Overview of the future market designs across Europe when all planned CRMs are implemented. Already today, the mechanisms are poorly coordinated, which might intensify due to additional mechanisms being established within the next few years. (Source reproduced from Bublitz et al. [2019], classification of mechanisms based on European Commission [2016])

\begin{tabular}{l|l}
\hline No CRM & De-central obligation \\
Strategic reserve & Tender for new capacity \\
Central buyer & Targeted capacity payments \\
\hline
\end{tabular}

and some Eastern European countries as well as Denmark and Italy. Different longterm simulations up to 2050 are carried out for all three REFLEX scenarios (ModRES, High-RES decentralized, High-RES centralized) to derive insights regarding the impact of national and coordinated CRM policies on amount and location of new investments, the resulting technology mixes in the electricity sector as well as generation adequacy.

\subsection{Research Design}

For the quantitative analyses on electricity market design carried out in the following, the agent-based simulation model PowerACE is applied. A brief overview of PowerACE is given in Chapter 3. Further model details can be found in the following references:

- Coupling and clearing of the day ahead markets (Ringler et al. 2017),

- Generation and storage expansion planning under consideration of cross-border effects (Fraunholz et al. 2019),

- Implemented capacity remuneration mechanisms (Keles et al. 2016). 


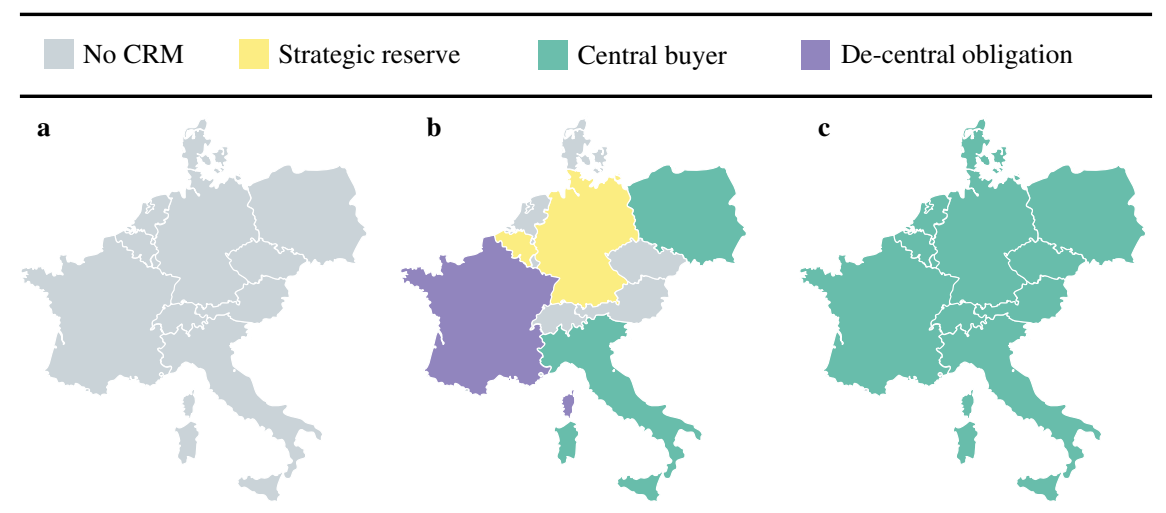

Fig. 11.2 Overview of the market areas modeled in PowerACE and their respective market design in the a "European EOM" setting, b "National CRM policies" setting, c "Coordinated CRM" setting. In order to capture a variety of different design options and corresponding cross-border effects, the regional scope covers Central Western European and some Eastern European countries as well as Denmark and Italy. (Source own illustration)

All three REFLEX scenarios (Mod-RES, High-RES decentralized, High-RES centralized $)^{2}$ are analyzed considering three different settings with regard to electricity market design (cf. Fig. 11.2):

- European EOM, which serves as a benchmark,

- National CRM policies, in which the market designs of each country as currently implemented or planned are considered, ${ }^{3}$

- Coordinated CRM, which describes a market design potentially standing better in line with the goals of creating an internal electricity market in Europe than unilateral CRMs.

The investment methodology in PowerACE depends on the respective market design. In market areas without an implemented CRM, the investment decisions are solely driven by the future electricity price expectations of the investors. Contrary, in market areas with an active central buyer mechanism, annual descending clock auctions are carried out in order to contract a specific amount of secured generation and storage capacity. For this purpose, the regulator first sets an arbitrary reserve margin, which controls the desired level of generation adequacy and defines the capacity to be procured in the auction.

\footnotetext{
${ }^{2}$ Detailed information on the different scenarios are provided in Chapter 2. For recapitulation: (i) the electricity demand grows moderately in Mod-RES and substantially higher in High-RES, (ii) significantly more intermittent renewables are assumed in High-RES than Mod-RES, (iii) decentralized solar power dominates High-RES decentralized, whereas higher shares of offshore wind power characterize High-RES centralized.

${ }^{3}$ Please note, that due to the similarities of the different types of CRMs on an abstract level, the French CRM is modeled using the central buyer implementation in PowerACE, although in reality, a de-central obligation mechanism is used in France.
} 
In case of uncoordinated national CRMs, the reserve margin $\mu_{y}^{\text {nat }}$ is set to 1.0 , such that the residual load in the respective market area can always be covered by the available conventional generation and storage capacity, without depending on electricity imports. The calculation of the required capacity $c_{m, y}^{\text {nat }}$ is shown in Eq. 11.1, where $d$ denotes the electricity demand, $r$ the renewable feed-in, $m$ the market area, $y$ the year, and $h$ the hour of the year.

$$
c_{m, y}^{\mathrm{nat}}=\mu_{y}^{\mathrm{nat}} \cdot \max _{h}\left(d_{m, y, h}-r_{m, y, h}\right) \quad \forall m, y
$$

For the coordinated CRM, the national reserve margin is adjusted by the ratio between peak residual load across all market areas and the sum of the national peak residual loads (cf. Equation 11.2). This procedure is also applied by Bucksteeg et al. (2019). Cross-border synergies obviously lead to lower reserve margins under the coordinated CRM $\mu_{y}^{\text {coor }}$ than in the uncoordinated case.

$$
\mu_{y}^{\text {coor }}=\mu_{y}^{\text {nat }} \cdot \frac{\max _{h} \sum_{m}\left(d_{m, y, h}-r_{m, y, h}\right)}{\sum_{m} \max _{h}\left(d_{m, y, h}-r_{m, y, h}\right)} \leq \mu_{y}^{\text {nat }} \quad \forall y
$$

Contrary to the model-endogenous investment decisions, decommissioning of existing power plants is exogenously defined in PowerACE and based on the respective age and technical lifetime of the generation units, which remain unchanged for all scenarios. Consequently, the development of the future technology mix across the various scenarios strongly depends on the techno-economic characteristics of the different investment options (conventional power plants and storage technologies). Since the expansion of RES is an exogenously defined and scenario-specific input to PowerACE, no additional investments in renewable technologies are considered. Moreover, the learning curves for storage technologies developed within the REFLEX project (cf. Chapter 4) are implemented in PowerACE.

\subsection{Development of the Conventional Generation Capacities and Wholesale Electricity Prices}

In the following, the simulation results for all three REFLEX scenarios are presented and discussed. The impact of the different market design settings on amount, location, and technology mix of new investments as well as the resulting wholesale electricity price developments is in the focus of the result presentation. The European EOM is used as a benchmark, to which the national CRM policies as well as the coordinated CRM setting are compared.

By imposing a certain capacity target and then offering payments to capacity providers additional to the income from selling electricity on the markets, CRMs tend to shift investment incentives in interconnected electricity markets toward the 
countries using such mechanisms. In the respective neighboring countries without an own CRM, investment incentives may stay stable, but could as well decrease due to the additional capacity from abroad, which also influences domestic price expectations of potential investors. Consequently, under national CRM policies, both positive and negative cross-border effects may be observed.

Contrary, in the case of a coordinated CRM, capacity targets are set for each country, which may result in stable investment incentives across all interconnected market areas. Moreover, as previously described, the total capacities required to secure generation adequacy are lower in a coordinated CRM, due to cross-border synergies and better balancing of fluctuating electricity production from RES.

With regard to wholesale electricity prices, according to theory, the introduction of CRMs should reduce the amount of scarcity situations and related peak prices and therefore result in lower electricity wholesale prices. However, under national CRM policies, suppressed investments in neighboring countries of those using a CRM may also lead to negative cross-border effects. Consequently, the implementation of a CRM might prove to be less effective as expected when considering only an isolated country.

A coordinated CRM should incentivize sufficient capacity to cover the electricity demand at all times and therefore reduce the wholesale electricity prices in all interconnected market areas. However, these savings come at the price of capacity payments for the additional capacity. These may to a certain extent compensate or even overcompensate the savings achieved by lower wholesale electricity prices. This effect-despite its high practical relevance-is however out of the scope of the work presented in this chapter and should be subject to future research.

Figures $11.3,11.4,11.5,11.6,11.7$ and 11.8 present the development of the total conventional generation and storage capacities as well as resulting wholesale electricity prices for all three REFLEX scenarios and two exemplary countries. ${ }^{4}$ Since all of these figures are structured similarly, some general remarks are provided before discussing the obtained results in more detail.

In the top part of the figures, the respective total conventional generation and storage capacities throughout the simulation period of 2020-2050 are shown for each of the three market design settings (European EOM, National CRM policies, Coordinated CRM-from left to right). Furthermore, the respective yearly national peak residual load, excluding imports/exports and storage is depicted as a reference point. As previously mentioned, the capacity developments are based on exogenously predefined decommissioning, which is identical for all investigated settings, as well as on model-endogenous investment decisions for different technologies.

The bottom part of the figures shows the resulting impact on the development of the wholesale electricity prices. For this purpose, the European EOM is defined as a reference and the relative price difference $\Delta p_{m, y}$ is then computed as the mean yearly

\footnotetext{
${ }^{4}$ Due to space limitations, only results for France and the Netherlands are presented in this section. These countries are chosen as representative ones in terms of cross-border effects, since France is using a CRM under the national CRM policies, while the Netherlands rely on an EOM, but are surrounded by countries applying some kind of CRM.
} 


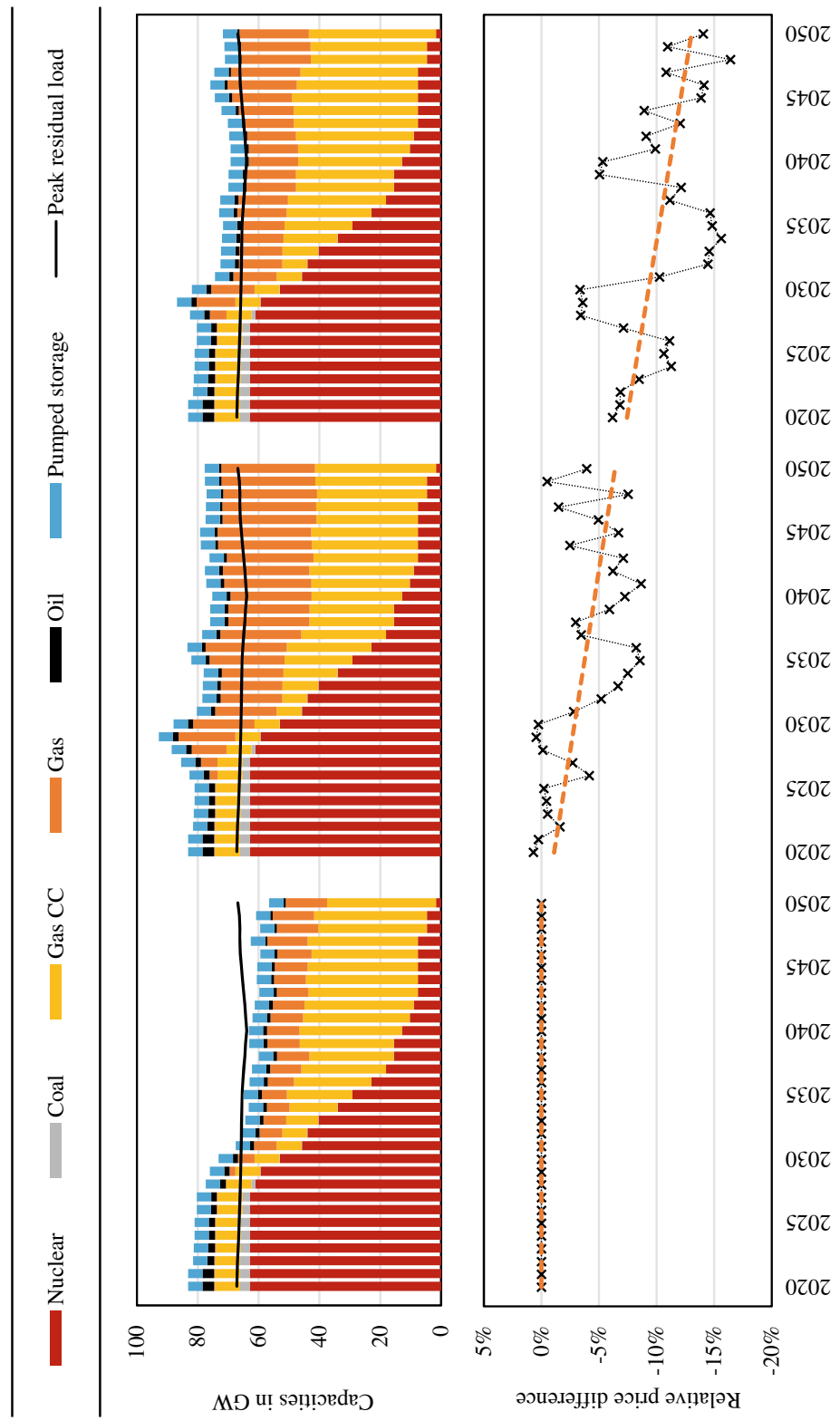

soz ๖. 크 व $\stackrel{0}{=}$ 可

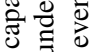

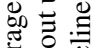
웅 웜

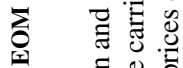

들

政

实苞节

过 퐁. . 氙 छ 울 雚 छे के 产 过 ค 등

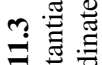
$\exists$ 覀 웅 
C. Fraunholz et al.
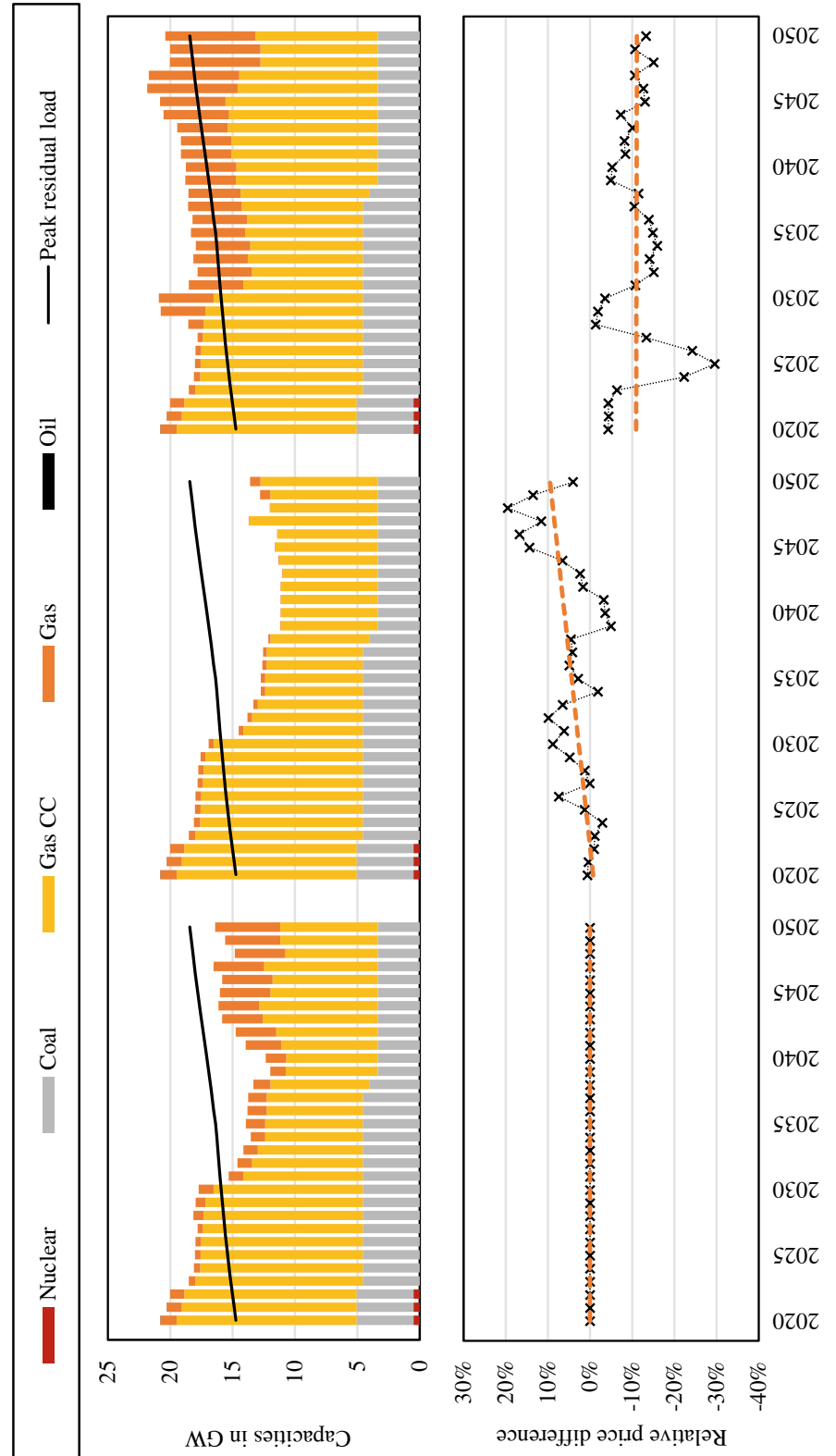

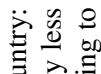

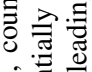
जิ 푱

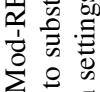
st0z
$0 t 0 z$
$\varsigma \varepsilon 0 z$
$0 \varepsilon 0 z$

$\because$ 이 해

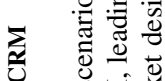

递

\&

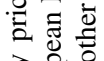

츨 을

szor 豞可

过 $\cong$

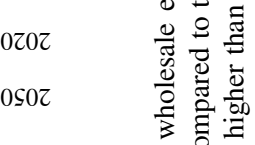

St0Z

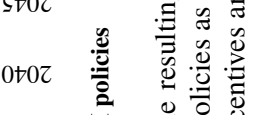

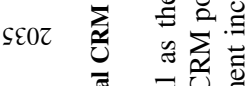

$0 \varepsilon 02$ 运

szoz 乙

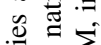

ozor

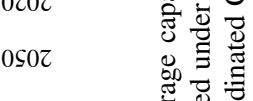

St0z क⿺辶

ot0z ¿

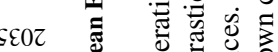

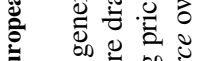

$0 \varepsilon 0 z$

至

szoz

$\cong$ 氙.

प艹

䒕 䒕

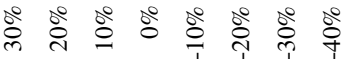

翓

리유

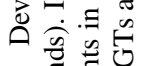

苯言芯记

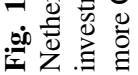




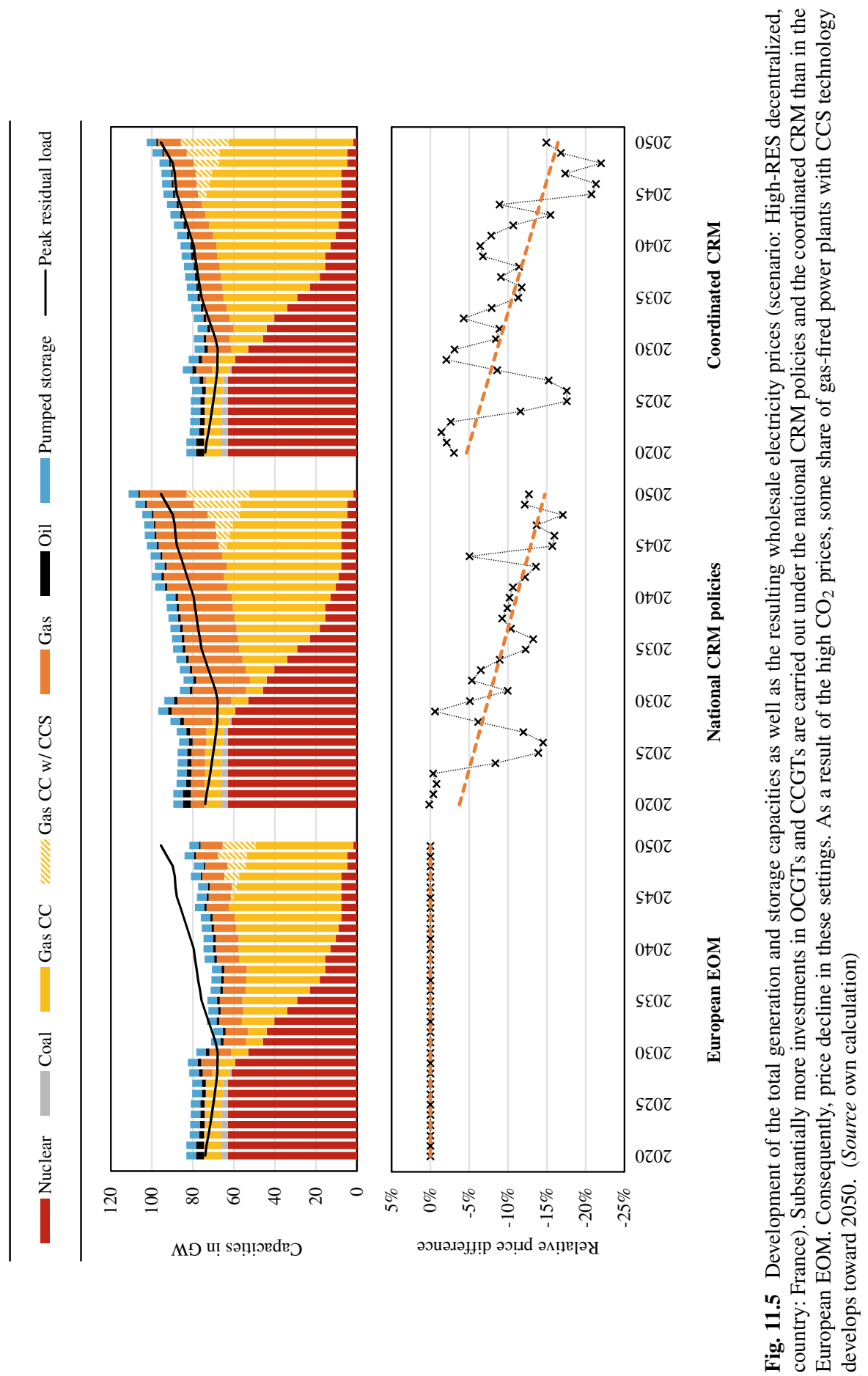




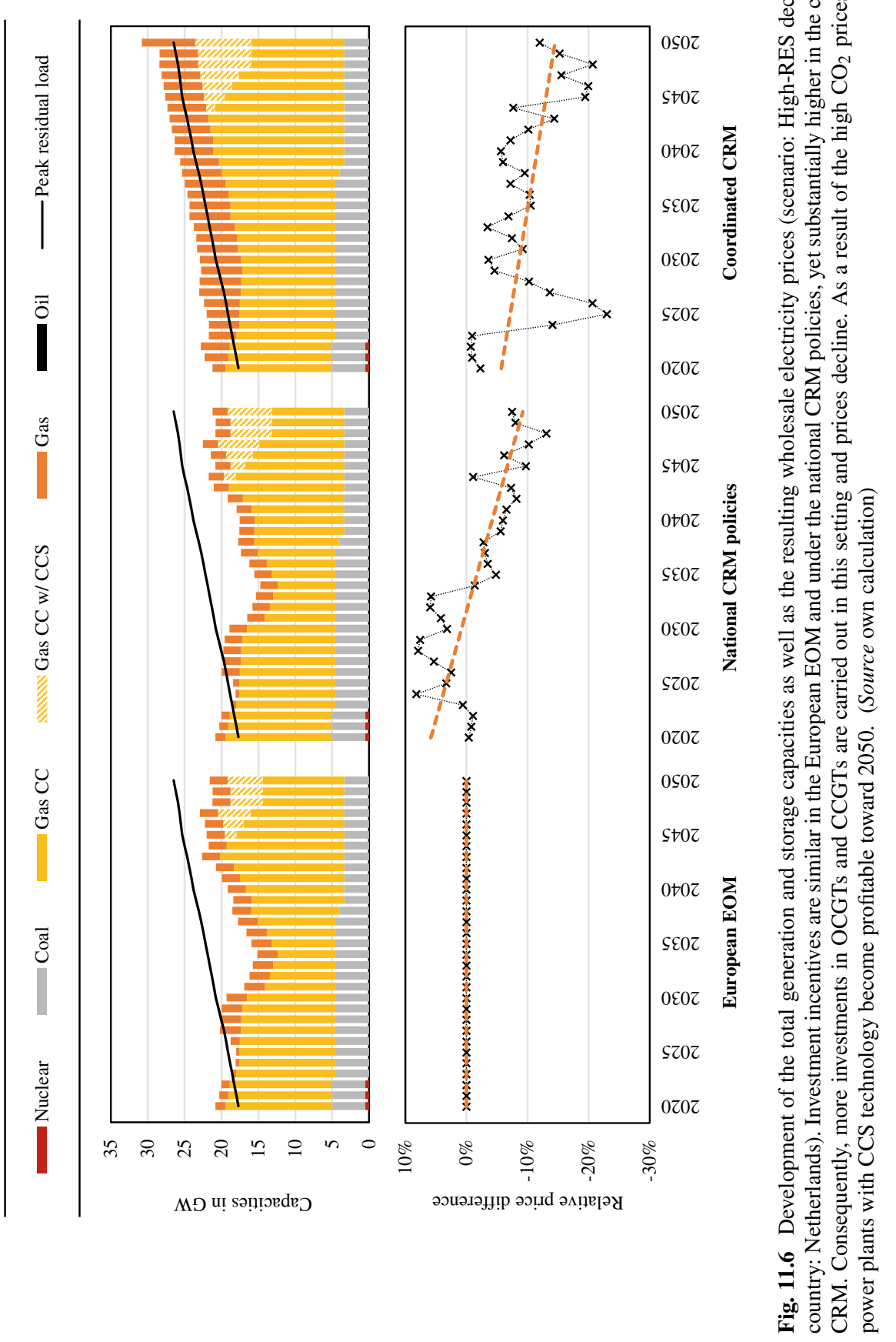



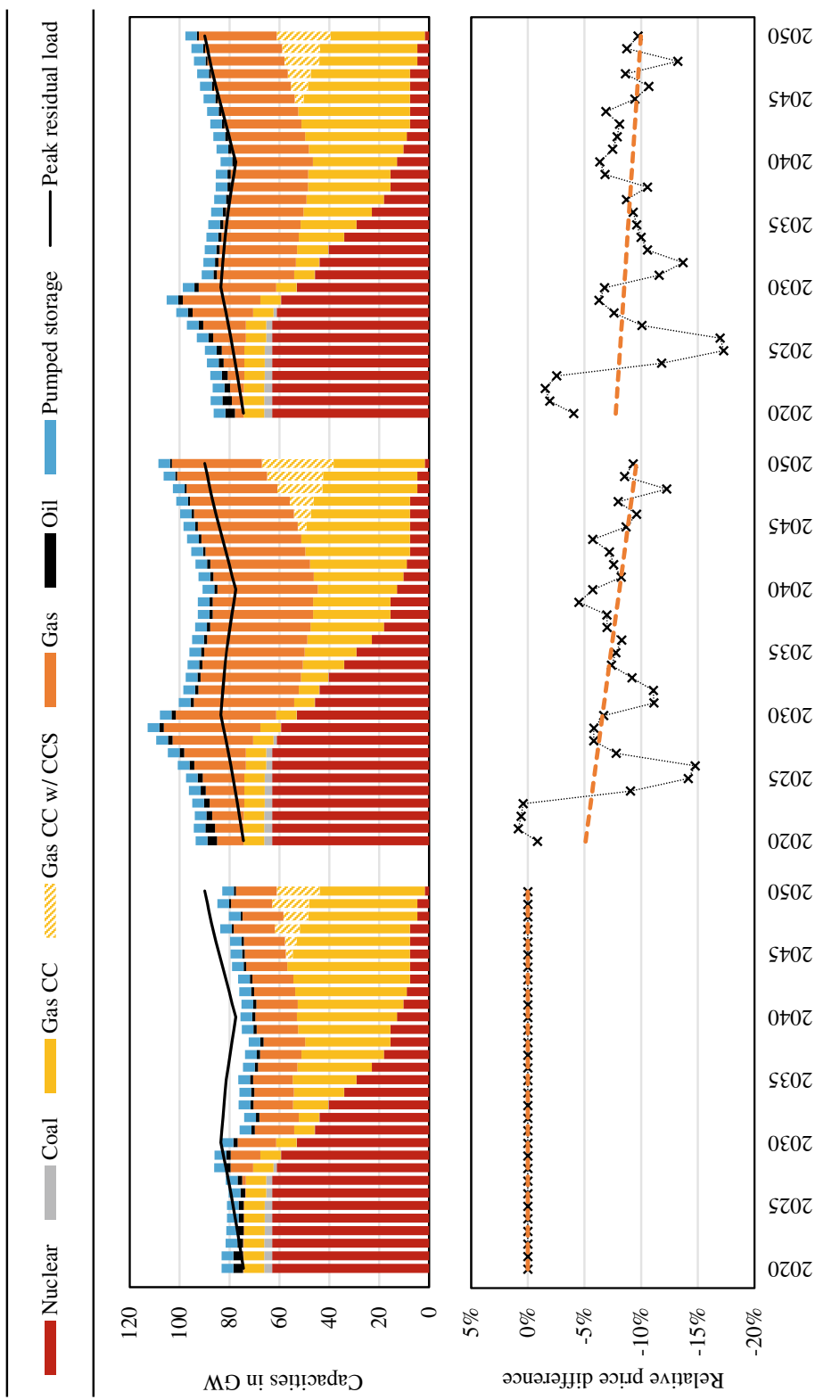

ชี สี

글 을 वृ.

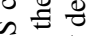
쇠시 立范

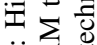
نَّ 


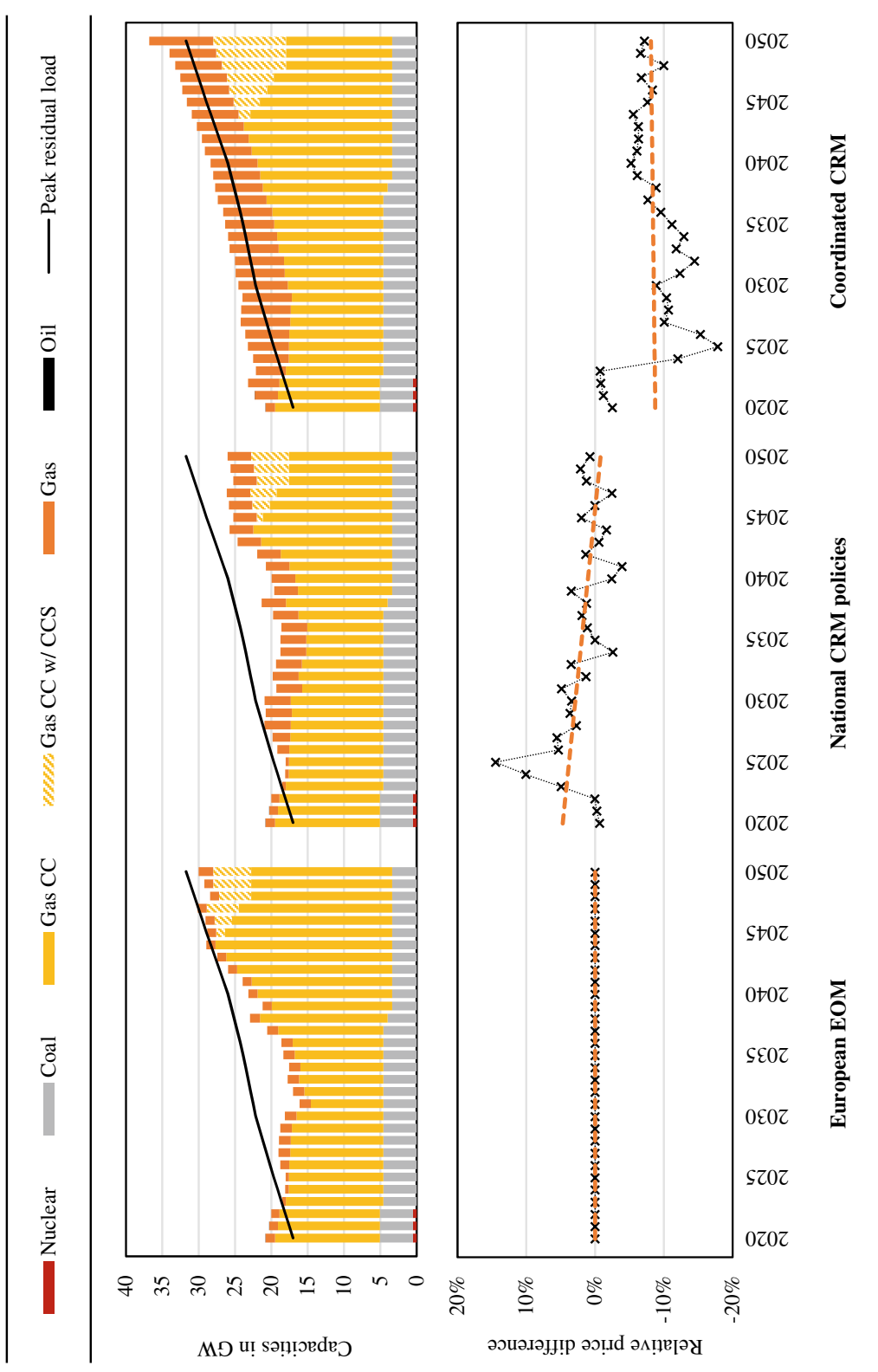

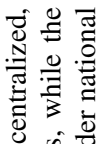

s.

늘 웜

定 $\sum$

$\ddot{0}$

프 픔

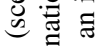

包 总

离

.气

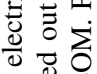

․․ㄹ 热

记

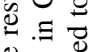

气 气

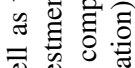

उ

政

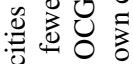

ใั ซี

ن :

啳

옹 일

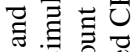

ธิ क छ

है

닝

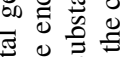

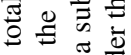

记芯焉

눙 总

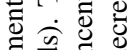

है.

율 $\sum^{\circ}$

ดั

$\infty$ 莌:

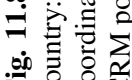


price under the respective market design $p_{m, y}^{*}$ divided by that under the European $\operatorname{EOM} p_{m, y}^{\text {ref }}$ as shown in Eq. 11.3, where $m$ denotes the market area and $y$ the year. Consequently, by definition, the relative price differences in the European EOM are always at $0 \%$ throughout the simulation period. For illustrative purposes, the linear trend of the yearly price differences is also included in the respective diagrams.

$$
\Delta p_{m, y}=\overline{p_{m, y}^{*}} / \overline{p_{m, y}^{\text {ref }}}-1 \quad \forall m, y
$$

In the following, some specific results of Mod-RES, High-RES decentralized, and High-RES centralized will be presented and discussed.

\subsubsection{Mod-RES Scenario}

In Mod-RES, some general trends across all modeled countries can be identified:

- A strong fuel switch toward gas-fired technologies, which is mainly driven by increasing $\mathrm{CO}_{2}$ prices,

- No investments in carbon capture and storage (CCS) technologies, due to an insufficiently high $\mathrm{CO}_{2}$ price development, which doesn't allow for costcompetitiveness of CCS in view of the higher initial investment and variable costs,

- Low profitability of storage investments, due to the moderate share of electricity generation from RES in Mod-RES. Moreover, the residual load curves provided to PowerACE as exogenous input data are already smoothed by demand side management (DSM) measures (for details cf. Chapters 3, 6, 7 and 8).

As presented in Fig. 11.3, substantially more investments in open cycle gas turbines (OCGT) are carried out in France under the national CRM policies than in the European EOM. This is a direct consequence of the French CRM, which successfully incentivizes additional peak capacity. To a lesser extent, this finding is also true under the coordinated CRM. Since the required reserve margin in a coordinated approach is lower than for a national CRM, less peak load capacity is built in this setting than under the national CRM policies.

The additional capacity has a direct impact on the development of the wholesale electricity prices, which decline under both the national CRM policies and the coordinated CRM as compared to the European EOM. Despite lower capacity levels in France, the price decline is more pronounced under the coordinated CRM. This effect is due to more capacity in the French neighboring countries under the coordinated CRM than under national CRM policies, from which also France seems to benefit.

In the Netherlands, which rely on an EOM, investment incentives are drastically reduced under the national CRM policies as compared to the European EOM. This finding can be attributed to negative cross-border effects caused by the Dutch neighboring countries using CRMs. 
Consequently, substantially less investments in peak load capacity, i.e., OCGTs are carried out under the national CRM policies, which results in increasing wholesale electricity prices (cf. Fig. 11.4). Contrary, in the coordinated CRM, investment incentives are higher than in both other market design settings due to a capacity target also for the Netherlands. This leads to additional OCGTs and lower prices.

\subsubsection{High-RES Decentralized Scenario}

Also in the High-RES decentralized scenario, some general trends across the modeled countries can be identified:

- A strong fuel switch toward gas-fired technologies, similarly as in Mod-RES, ${ }^{5}$

- Substantial investments in CCS technologies toward the end of the simulation period, which is a result of the higher $\mathrm{CO}_{2}$ prices than in Mod-RES,

- Low profitability of storage investments, for similar reasons as in Mod-RES. Given the strong increase in electricity demand, electricity generation from RES remains moderate in relative terms despite its significant increase in absolute figures. Consequently, investments in OCGTs remain more profitable than storage investments even in the long run. Furthermore, the residual load curves have again been substantially smoothed by DSM measures prior to their use in PowerACE (for details cf. Chapters 3, 6, 7 and 8).

As shown in Fig. 11.5, similarly as in Mod-RES, substantially more investments in OCGTs are carried out in France under the national CRM policies and the coordinated CRM than in the European EOM. However, due to the significant increase in electricity demand and the substantial smoothing of the load curve through DSM measures, the French CRM also incentivizes additional combined cycle gas turbines (CCGT). As a result, the wholesale electricity prices are lower under both the national CRM policies and the coordinated CRM as compared to the European EOM.

Owing to cross-border effects of the CRMs in neighboring countries, the investment incentives in the Netherlands are reduced under the national CRM policies also in High-RES decentralized. Yet, contrary to Mod-RES, these effects are much less pronounced. This is due to the strongly increasing demand across all countries in High-RES decentralized, which leads to a relatively high number of running hours for new capacity and therefore CCGTs often being the more profitable investment option than additional OCGTs. Since CRMs mainly affect the allocation of peak load capacity, i.e., OCGTs, the amount of investments in countries without CRM is less affected by cross-border effects of the national CRM policies in High-RES decentralized than in Mod-RES.

\footnotetext{
${ }^{5}$ Although the $\mathrm{CO}_{2}$ prices are assumed to grow stronger in High-RES decentralized than in Mod-RES, some coal-fired generation remains in the market even in 2050. This is because decommissioning of power plants is only considered exogenously based on their respective age.
} 
In the coordinated CRM, for similar reasons as in France, substantial amounts of additional investments in both OCGTs and CCGTs are carried out. These capacity developments also affect the wholesale electricity prices in the Netherlands (cf. Fig. 11.6). Due to the small reduction in domestic capacities under the national CRM policies and the additional capacity in the neighboring countries, prices decrease even under this setting in the long run as compared to the European EOM. This effect is even more pronounced under the coordinated CRM.

\subsubsection{High-RES Centralized Scenario}

The general trends regarding fuel switch and profitability of CCS and storage technologies, which could be identified in the results of High-RES decentralized, also apply to the High-RES centralized scenario. Regarding the development of capacity levels and wholesale electricity prices, the patterns in the High-RES centralized scenario are quite similar to those of High-RES decentralized in France. However, due to the significantly lower amount of DSM measures in the High-RES centralized scenario (cf. Chapters 2, 6, 7 and 8), more OCGTs and less CCGTs are built (cf. Fig. 11.7).

In the Netherlands, less CCGTs and more OCGTs are built under the national CRM policies than in the European EOM (cf. Fig. 11.8). This is likely due to a reduced number of running hours for new capacity caused by the additional capacity incentivized in the Dutch neighboring countries due to their CRMs. In the coordinated CRM, the patterns are very similar to those in the High-RES decentralized scenario. Regarding the development of the wholesale electricity prices, no clear trend can be identified under the national CRM policies, while prices decline in the coordinated CRM as compared to the European EOM.

\subsection{Impact on Generation Adequacy}

Generation adequacy can be defined as the ability of an electricity system to provide sufficient dispatchable generation, storage, and flexible demand side capacity to cover the residual load at any time. Since the electrical grid is not modeled in PowerACEapart from the simplified consideration of maximum cross-border transmission capacities-grid restrictions are not considered in the evaluation of the generation adequacy presented in the following. Yet, the focus of this work is rather on the ability of different electricity market designs to provide adequate investment incentives to achieve a sufficient capacity level under consideration of the respective cross-border effects.

In order to assess and compare generation adequacy across the various settings and for all modeled countries, a simple, but straightforward indicator is applied. In the investment methodology of PowerACE, no restriction to cover the demand 
at all times is implemented, but the expansion planning rather emerges from the individual actors' decisions (cf. Chapter 3). Thus, situations may occur in which the day ahead market cannot be cleared due to an insufficient level of dispatchable generation and storage capacity, leading to the maximum day ahead market price of 3,000 EUR/MWh. In reality, reserve capacity would likely be activated, such that even in these scarcity situations, load shedding would not necessarily occur. Yet, the mean number of yearly hours with no successful clearing of the day ahead market in a given market area is a suitable proxy to measure generation adequacy.

Figure 11.9 provides a concise overview of the generation adequacy levels in all scenarios, market design settings, and countries. Across all scenarios, the unilateral implementation of CRMs under the national CRM policies rather obviously increases generation adequacy in the respective countries. However, also neighboring countries of those using a CRM seem to benefit from the additional capacity and face an increase in their generation adequacy levels. This finding indicates that free riding occurs. The effect is most pronounced in High-RES decentralized. As described before, the strongly growing electricity demand in this scenario combined with the extensive use of DSM measures leads to CCGTs often being more profitable than OCGTs. However, since CRMs mainly shift investment incentives for peak load capacity, investments in countries without a CRM barely decline in this scenario. Thus, these countries benefit from an almost unchanged level of domestic capacity plus the additional capacity of their neighbors with CRMs.

Interestingly, generation adequacy increases even further under a coordinated CRM, even in the countries that already use a CRM under the national CRM policies. Apparently, cross-border synergies, better balancing of fluctuating electricity production from RES as well as reduced free riding by neighboring countries without an own CRM, outweighs the impact of lower domestic capacity levels in the respective countries under the coordinated approach.

\subsection{Summary and Conclusions}

In this chapter, the electricity market model PowerACE was applied to a region covering multiple interconnected European market areas with different electricity market designs. Several long-term simulations up to 2050 were carried out for all three REFLEX scenarios (Mod-RES, High-RES decentralized, High-RES centralized) in order to quantitatively assess the long-term cross-border effects of CRMs in the European electricity system. In this context, three different settings with regard to electricity market design were analyzed. Firstly, a European EOM, which served as a benchmark. Secondly, national CRM policies, including the unilateral introduction of CRMs as currently planned or already implemented in reality. Thirdly, a coordinated CRM as an approach potentially standing better in line with the goals of creating an internal electricity market in Europe than unilateral CRMs. By comparing the different settings, valuable insights regarding the impact of national and coordinated CRMs on amount and location of new investments, the resulting technology mixes 


\begin{tabular}{llll|l|l}
\hline $0 \mathrm{~h} / \mathrm{a}$ & $1-3 \mathrm{~h} / \mathrm{a}$ & $4-10 \mathrm{~h} / \mathrm{a}$ & $11-15 \mathrm{~h} / \mathrm{a}$ & $>15 \mathrm{~h} / \mathrm{a}$ \\
\hline
\end{tabular}
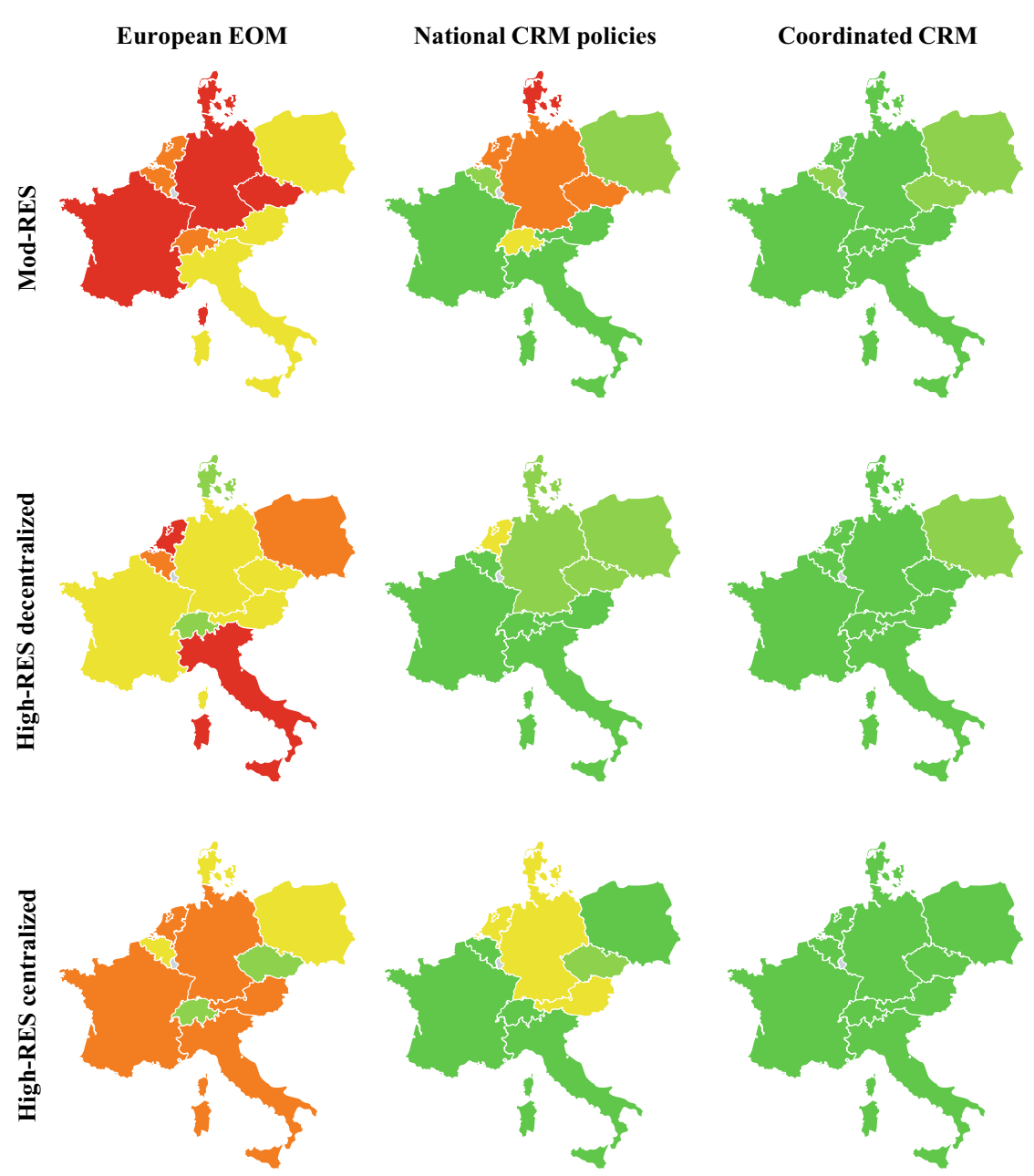

Fig. 11.9 Mean yearly hours with no successful clearing of the day ahead market, i.e., the maximum day ahead market price of 3,000 EUR/MWh due to a shortage of dispatchable generation and storage capacity. All values are averaged over the years 2020 to 2050 and given in h/a. Across all scenarios, the implementation of CRMs does not only increase generation adequacy in the countries using the CRMs, but also in their neighboring countries. (Source own calculation)

in the electricity sector, the development of the wholesale electricity prices as well as generation adequacy could be derived.

In terms of the future technology mix, across all investigated scenarios and market areas, a strong fuel switch toward gas-fired power plants can be observed as a result of the assumed $\mathrm{CO}_{2}$ price development. Due to the more extreme assumptions with 
regard to $\mathrm{CO}_{2}$ prices in the High-RES scenarios, $\mathrm{CCS}$ technologies turn out to be profitable toward 2050, while this is not the case in the Mod-RES scenario. Furthermore, in all scenarios, storage technologies only play a minor role under the assumptions made. This finding is related to the moderately ambitious shares of renewable electricity generation, even in the High-RES scenarios due to the strongly increasing electricity demand. Besides, the applied electricity load curves are already smoothed by DSM measures prior to their implementation in PowerACE.

The unilateral introduction of CRMs proves to be an effective measure substantially shifting investment incentives toward the countries implementing the mechanisms. The additional generation capacity in these countries in turn reduces both the average wholesale electricity prices and the amount of scarcity situations. Depending on the specific setting, neighboring countries of those implementing a CRM may face both positive and negative cross-border impacts.

In the Mod-RES scenario, which is characterized by a moderate growth of electricity demand, OCGTs often prove to be the most profitable investment option. However, building more peak load capacity in countries with an active CRM drastically reduces investment incentives in neighboring countries without an own CRM, leading to increasing wholesale electricity prices in these countries.

Contrary, in the High-RES scenarios, where the electricity demand grows stronger over time, investments in CCGTs are often economically preferable over peak load capacity. Yet, in contrast to OCGTs, the profitability of CCGTs in countries without an own CRM is less affected by additional investments in neighboring countries with CRMs. Consequently, in the long run, the average wholesale electricity prices may decrease also in countries without an own CRM.

Despite the distortion of investment incentives, across all scenarios, CRMs generally increase generation adequacy not only in the country implementing the mechanism, but also in the neighboring countries, indicating that free riding occurs.

In all three scenarios, a coordinated CRM, in which capacity targets are set for each individual country, provides adequate investment incentives in all countries. Although individual capacity requirements are lower than in case of an unilateral introduction of a CRM, all countries benefit in terms of lower wholesale electricity prices and increased generation adequacy levels. This is also true for countries that already use a CRM under the national CRM policies setting. Apparently, reduced free riding by neighboring countries without an own CRM outweighs the impact of lower domestic capacity levels on wholesale electricity prices in the respective countries under the coordinated approach. However, the savings in terms of wholesale electricity prices come at the price of capacity payments for the additional capacity. These may to a certain extent compensate or even overcompensate the savings achieved by lower wholesale electricity prices. This effect was not considered in this chapter, but should be subject to future research in order to get a holistic picture.

Summing up, whether positive or negative cross-border effects of unilateral CRMs prevail, depends on a variety of factors, including the future development of electricity demand and renewable electricity generation as well as the geographical location of a given country. A coordinated approach generally seems preferable in terms of wholesale electricity prices and generation adequacy. The European Commission 
should therefore continue to assess potential CRMs carefully prior to allowing their real-world implementation and consider a coordinated European CRM as an alternative market design solution potentially standing better in line with the goals of creating an internal electricity market in Europe.

Although the analyses presented in this chapter provide valuable insights regarding long-term cross-border effects of CRMs in the European electricity system, open questions for future research remain. Two aspects of particular relevance are as follows. Firstly, the role of electricity storage would likely become more visible in a modeling approach where DSM measures simultaneously compete with storage technologies rather than smoothing the electricity load curves prior to their implementation in an electricity market model like PowerACE. DSM measures could then also participate in the CRMs in the same fashion as storage technologies. Secondly, in a real-world setting, also interconnector capacities are typically allowed to participate in CRMs of neighboring countries. Considering this aspect would probably reduce the cross-border effects of unilateral CRMs as presented in this chapter and therefore bring the situation closer to that of a coordinated European CRM, yet at a lower administrative burden.

\section{References}

Bublitz A, Keles D, Zimmermann F, Fraunholz C, Fichtner W (2019) A survey on electricity market design: insights from theory and real-world implementations of capacity remuneration mechanisms. Energy Econ 80:1059-1078. https://doi.org/10.1016/j.eneco.2019.01.030

Bucksteeg M, Spiecker S, Weber C (2019) Impact of coordinated capacity mechanisms on the European power market. Energy J 40:221-264. https://doi.org/10.5547/01956574.40.2.mbuc

European Commission (2016) Commission staff working document on the final report of the sector inquiry on capacity mechanisms: SWD (2016) 385 final, Brussels. https://ec.europa.eu/energy/ sites/ener/files/documents/swd_2016_385_f1_other_staff_working_paper_en_v3_p1_870001. pdf

Fraunholz C, Keles D (2019) D5.2 Report on investments in flexibility options considering different market designs. Report for the REFLEX project

Fraunholz C, Keles D, Fichtner W (2019) Agent-based generation and storage expansion planning in interconnected electricity markets. In: 2019 16th International Conference on the European Energy Market (EEM). https://doi.org/10.1109/EEM.2019.891634.

Keles D, Bublitz A, Zimmermann F, Genoese M, Fichtner W (2016) Analysis of design options for the electricity market: the German case. Appl Energy 183:884-901. https://doi.org/10.1016/ j.apenergy.2016.08.189

Ringler P, Keles D, Fichtner W (2017) How to benefit from a common European electricity market design. Energy Policy 101:629-643. https://doi.org/10.1016/j.enpol.2016.11.011 
Open Access This chapter is licensed under the terms of the Creative Commons Attribution 4.0 International License (http://creativecommons.org/licenses/by/4.0/), which permits use, sharing, adaptation, distribution and reproduction in any medium or format, as long as you give appropriate credit to the original author(s) and the source, provide a link to the Creative Commons license and indicate if changes were made.

The images or other third party material in this chapter are included in the chapter's Creative Commons license, unless indicated otherwise in a credit line to the material. If material is not included in the chapter's Creative Commons license and your intended use is not permitted by statutory regulation or exceeds the permitted use, you will need to obtain permission directly from the copyright holder.

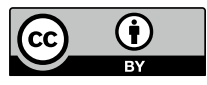

